\title{
Sonographic Biometry of the Frontal Lobe in Normal and Growth-Restricted Neonates
}

\author{
IMAD R. MAKHOUL, MICHALLE SOUDACK, ISRAEL GOLDSTEIN, TATIANA SMOLKIN, \\ ADA TAMIR, AND POLO SUJOV \\ Department of Neonatology, Meyer Childrens Hospital [I.R.M., T.S., P.S.], and Departments of Diagnostic \\ Imaging [M.S.], Obstetrics and Gynecology [I.G.], and Community Medicine [A.T.], Rambam Medical \\ Center, and the Bruce Rappaport Faculty of Medicine, Technion-Israel Institute of Technology, Bat-Galim,
} Haifa 31096, Israel.

\begin{abstract}
Assessing the impact of restricted intrauterine growth on neonatal frontal lobe (FL) dimensions is important. We aimed to create a sonographic nomogram of FL dimensions in neonates at different gestational ages (GA) and evaluate the impact of small head circumference (HC) on FL dimensions. We conducted sonographic biometry of the FL at birth. We included 218 newborn infants born at GA of 24-43 wk: appropriate for GA and normal HC $(n=178)$, and small for GA and small HC ( $n=$ 23). Infants with a 5-min Apgar score $<7$, severe congenital malformations, or chromosomal abnormalities were excluded. Through a coronal ultrasound scan via the anterior fontanelle at the level where the most lateral point of the left Sylvian fissure was best demonstrated, we drew a triangle connecting the most lateral point of the Sylvian fissure, the corpus callosum, and the subcalvarian point of the interhemispheric fissure. We measured the three sides of the triangle, Sylvian-fontanellar distance, Sylvian-callosal distance, and fontanellar-callosal distance, and calculated the frontal triangular area. All four FL dimensions increased significantly between 24 and 43 wk of gestation in both appropriate for GA-normal $\mathrm{HC}$ and small for GA-small $\mathrm{HC}$ neonates, and were strongly correlated with $\mathrm{HC}$ and birth weight. Regression lines of GA against Sylvian-fontanellar distance, Sylvian-callosal distance, fontanellar-callosal distance, and fron-
\end{abstract}

\section{ABSTRACT}

tal triangular area in the appropriate for GA-normal HC group differed significantly from those of the small for GA-small HC group $(p<0.05)$. Male neonates had significantly larger Sylvianfontanellar and Sylvian-callosal distances than females $(p<0.01$ and $p<0.015$, respectively). In conclusion, FL measures increased significantly between 24 and 43 wk of gestation, and were strongly correlated with $\mathrm{HC}$. We speculate that a sonographically small fetal $\mathrm{HC}$ implies growth restriction of the fetal FL. (Pediatr Res 55: 877-883, 2004)
AGA, appropriate for gestational age
FCD, fontanellar-callosal distance
FL, frontal lobe
FTA, frontal triangular area
GA, gestational age
HC, head circumference
IUGR, intrauterine growth restriction
MRI, magnetic resonance imaging
SCD, Sylvian-callosal distance
SFD, Sylvian-fontaneller distance
SGA, small for gestational age
US, ultrasonography

The FL constitutes approximately one third of the cerebral hemisphere. Growth of the FL is essential for achieving normal cognitive functions and intelligence (1-4). Previous US studies have shown that FL hypoplasia and microcephaly in the fetus are very much interrelated (5-10), and that delayed growth of the fetal FL is significantly associated with fetal microcephaly (10). Furthermore, a shortened or small FL on fetal US has been suggested as a marker for fetal trisomy $21(5,7,9)$.

US biometry of the FL in the fetus has been previously investigated and is well-described in the medical literature (5, 7-10). To the best of our knowledge, however, there are no

Received July 21, 2003; accepted December 11, 2003.

Correspondence: Imad R Makhoul, M.D., D.Sc., Department of Neonatology, Rambam Medical Center, Bat-Galim, Haifa 31096, Israel; e-mail: makhoul@rambam.health.gov.il

DOI: 10.1203/01.PDR.0000119369.21770.7A reports of US FL biometry in neonates. Previous US studies of the fetal head (axial scans) were based on three different measurements for the estimation of the fetal FL size: 1) from the inner calvaria to posterior cavum septum pellucidum (8);2) frontothalamic distance, i.e. from the frontal bone to posterior edge of thalamus $(5,7,9,10)$; and 3) FL distance, i.e. from the anterior edge of the frontal horns of the lateral ventricles to the frontal bone (10).

FL hypoplasia in the fetus might cause delayed growth of the fetal head and could result in microcephaly and mental retardation (11). We assumed that the US dimensions of the neonatal FL at birth reflect those of a fetus at the same GA, and that they will be influenced by GA and HC. Therefore, we developed a novel model for US biometry of the FL and established a nomogram of neonatal FL dimensions across 
various GAs. We also evaluated the impact of fetal growth restriction and microcephaly on the FL growth profile.

\section{PATIENTS AND METHODS}

Serial US scans of the neonatal brain for ruling out hemorrhage are routine procedures for all premature newborn infants hospitalized in our neonatal intensive care unit at Meyer Children's Hospital, Haifa, Israel. Therefore, obtaining parental informed consent was not necessary.

Study population. The study population consisted of 218 newborn infants who were hospitalized in the neonatal intensive care unit for more than $24 \mathrm{~h}$, and were born between 24 and 43 wk of gestation at Rambam Medical Center between November 1, 2001, and December 31, 2002. Infants with a 5 -min Apgar score $<7$, severe congenital malformations, or chromosomal abnormalities were excluded. GA was calculated based on the date of the last menstrual period, early first trimester US data, and measurement of transcerebellar distance after birth (12). Dubowitz examination was not performed.

Of 218 studied neonates, 62 were term neonates and 156 were preterm neonates. Table 1 demonstrates the demographic and perinatal characteristics of the study population. Most of the studied neonates were singleton infants, AGA, normal HC, and delivered by head presentation.

Study groups. HC was measured at $24 \mathrm{~h}$ of life to minimize the impact of cranial s.c. edema, often seen after birth, on HC measurement. AGA status and normal $\mathrm{HC}$ were defined as birth weight and $\mathrm{HC}$ values, respectively, between the third and 97th centiles (13). SGA and small HC were defined as birth weight and $\mathrm{HC}<$ third centile (13). These definitions are widely used by most neonatologists. The 218 studied neonates

Table 1. Demographic and perinatal data of all 218 examined neonates

\begin{tabular}{lc}
\hline \multicolumn{1}{c}{ Variable } & $n=218$ \\
\hline Gestational age (wk) & $34.63 \pm 3.98$ \\
Mean & $24-43$ \\
Range & \\
Birth weight (g) & $2207 \pm 833$ \\
Mean & $500-4515$ \\
Range & $31(14.2 \%)$ \\
SGA & $183(83.9 \%)$ \\
AGA & $4(1.8 \%)$ \\
LGA & \\
Head circumference (cm) & $28(12.8 \%)$ \\
Microcephaly & $190(87.2 \%)$ \\
Normocephaly & $117(53.7 \%)$ \\
Sex & $101(46.3 \%)$ \\
Male & \\
Female & $94(43.1 \%)$ \\
Mode of delivery & $124(56.9 \%)$ \\
Spontaneous vaginal & \\
Cesarean section & $173(79.4 \%)$ \\
Presentation & $45(20.6 \%)$ \\
Head & \\
Breech & $170(78.0 \%)$ \\
Plurality & $48(22.0 \%)$ \\
Singletons & $1.06 \pm 0.96$ \\
Multiple births & \\
Age at US examination (day) & \\
\hline
\end{tabular}

Abbreviation used: LGA, large for GA. were distributed as follows: 178 AGA-normal HC, 23 SGAsmall HC, eight SGA-normal HC, five AGA-small HC, and four large for GA-normal $\mathrm{HC}$ neonates.

FL measurements. The US examinations were performed with a 128-XP scanner (Acuson, Mountain View, CA, U.S.A.) and a 5- to $7-\mathrm{MHz}$ linear transducer. Between 4 and $36 \mathrm{~h}$ of life, we scanned the left FL in each neonate. A coronal scan was obtained via the anterior fontanelle at the level where the most lateral point of the left Sylvian fissure was best demonstrated (Fig. 1). The precision of the electronic scale was 0.1 $\mathrm{mm}$.

Using electronic calipers we created a triangle connecting the most lateral point of the Sylvian fissure, the corpus callosum where it crosses the interhemispheric fissure, and the subcalvarian point of the interhemispheric fissure (hereafter, fontanelle) and measured its three sides: SFD, SCD, and FCD, and then calculated the FTA:

$$
\text { FTA }=0.25 \times \sqrt{\left(2 a^{2} b^{2}+2 b^{2} c^{2}+2 a^{2} c^{2}-a^{4}-b^{4}-c^{4}\right)}
$$

where $a, b$, and $c$ represent the sides of the triangle (Fig. 1).

Three of the authors (I.R.M., M.S., P.S.) conducted a preliminary pilot study and performed US measurements in a small group of infants (seven AGA neonates, results not included). Consensus was achieved regarding scanning consistency and the identification of intracranial landmarks, as the inter- and intraobserver error was $0-1 \mathrm{~mm}$.

Statistical analysis. Statistical analysis was performed using SPSS (SPSS Inc., Chicago, IL, U.S.A.). To detect variables with independent effect on each US dimension of the FL, univariate analysis was performed for each of the following perinatal variables: GA, birth weight, sex, presentation, mode of delivery, and plurality. Only variables with statistical significance of $<0.1$ were then included in the multiple regression analysis model (14). Because of the very strong statistical relationship between $\mathrm{HC}$ and each of the FL dimensions, $\mathrm{HC}$ was excluded from the regression analysis. Comparison of the slopes and intercepts of regression lines among the study groups was assessed by regression analysis. A $p$ value of less than 0.05 was considered statistically significant.

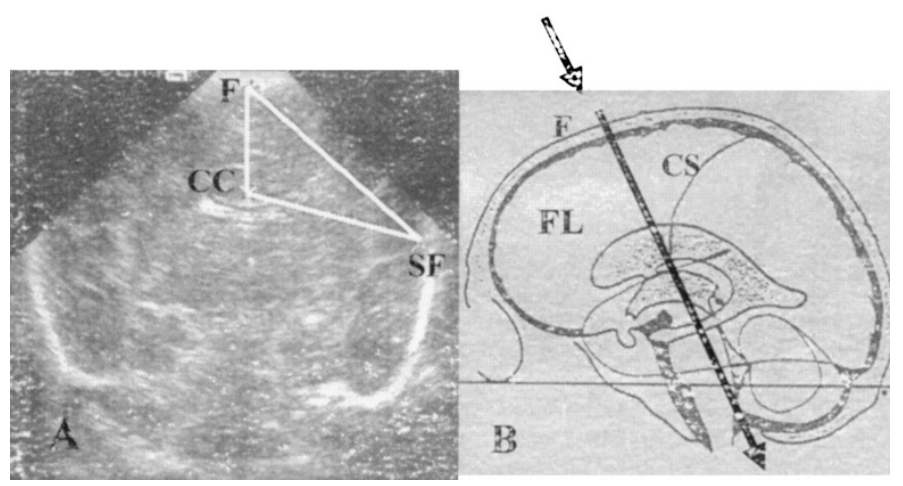

Figure 1. $A$, coronal scan via the anterior fontanelle $(F)$ : triangle formed by the most lateral point of the Sylvian fissure $(S F)$, the corpus callosum $(C C)$, and the subcalvarian point of the interhemispheric fissure. $B$, localization diagram showing the plane (arrow) of US scan of the frontal lobe via anterior fontanelle. (CS, central sulcus.) 


\section{RESULTS}

\section{FL dimensions in the study groups}

AGA-normal HC neonates. Table 2 demonstrates the mean values of frontal lobe US dimensions (SFD, SCD, FCD, FTA) among the AGA-normal HC group across various gestational weeks. Significant linear growth functions $(p<0.001)$ were found between GA and SFD (Fig. 2A), SCD (Fig. 3A), FCD (Fig. 4A), and FTA (Fig. 5A). In addition, significant linear growth functions $(p<0.001)$ were found between $\mathrm{HC}$ and SFD (Fig. $2 B$ ), SCD (Fig. $3 B$ ), FCD (Fig. $4 B$ ), and FTA (Fig. $5 B$; Table 3).

SGA-small HC neonates. Significant linear growth functions $(p<0.001)$ were found between GA and SFD (Fig. 2A), SCD (Fig. 3A), FCD (Fig. 4A), and FTA (Fig. 5A). In addition, significant linear growth functions $(p<0.001)$ were found between HC and SFD (Fig. 2B), SCD (Fig. 3B), FCD (Fig. 4B), and FTA (Fig. 5B; Table 3).

Comparison of study groups. The AGA-normal HC group and the SGA-small HC group differed significantly $(p<0.05)$ regarding the slopes and the intercepts of the regression lines of GA against SFD, SCD, FCD, or FTA (Figs. 2-5). However, these two groups were not statistically significantly different as to the slopes and the intercepts of the regression lines of $\mathrm{HC}$ against SFD, SCD, FCD, or FTA.

\section{Perinatal variables and FL dimensions}

Table 4 shows the results of the regression analysis model that included three perinatal variables that were significant in the univariate analysis: GA, birth weight, and sex. All four FL dimensions were significantly correlated with birth weight. Except for FCD, the other three FL dimensions were independently significantly correlated with GA. In addition, SFD and SCD were significantly larger in male compared with female neonates. Mode of delivery, presentation, and plurality were not statistically correlated with FL dimensions.

\section{DISCUSSION}

Our results show that the dimensions of the FL (SFD, SCD, FCD, and FTA), as measured by US after birth, increased significantly during the course of gestation and were strongly correlated with HC in both AGA-normal HC and SGA-small
HC neonates. These two groups differed significantly as to the regression lines of GA against each of the four studied FL measurements. In addition, male neonates had significantly larger SFD and SCD than female neonates. Neonatal head shape had no effect on all four measured FL sonographic variables, as evidenced by lack of significant effect of fetal presentation on these measurements.

Besides US, other imaging techniques have been used for measuring the volume of the FL. These included quantitative MRI (1, 15), three-dimensional MRI (16-20), singlephoton emission computed tomography (2), and computed tomography of the brain that measured the frontal force index (11). The temporal lobes have also been examined by US in infants by Sasaki et al. (21); however, their study described the optimal coronal plane for the visualization rather than for assessment of the growth profile of these lobes. Although MRI of the brain might offer some advantages over US in terms of better assessment of FL volume and white matter thickness, MRI is impractical as a bedside screening modality in neonatal units.

The SGA-small HC neonates (body weight and $\mathrm{HC}<$ third percentile) in our study had significantly smaller SFD, SCD, FCD, and FTA than their AGA-normal HC mates, across GA. It appears that fetuses who experienced severe IUGR commencing before 32 wk gestation already had growth-restricted FL. This is contrary to the reported growth profile of the fetal cerebellum (12), in which $60.7 \%$ of the SGA-small HC neonates had a preserved cerebellar width at birth. Hence, contrary to the cerebellum, it appears that the FL is more affected by slowed head growth in the fetus. Further studies are needed to assess the vulnerability of other cerebral lobes to severe IUGR.

Our observations regarding the smaller FL dimensions of the SGA-small HC neonates are in agreement with the reports on delayed growth of the FL in fetuses with severe IUGR and microcephaly (10). Additional conditions that were reportedly associated with a small or slow-growing FL in infants and children include trisomy $21(5,7,9)$, epilepsy (1), severe mental retardation (11), and malnutrition (17). In adults with schizophrenia, MRI showed a reduced volume of FL, with a corresponding increase in the volume of sulcal fluid (15). Also, FL dysplasia in adults was found to be associated with lower intelligence and epileptic attacks (2). Women with fragile-X gene syndrome were found to have FL dysfunction expressed

Table 2. Sonographic measurements of the left FL of AGA-normal HC neonates*

\begin{tabular}{crcccc}
\hline Gestational age $(\mathrm{wk})$ & $n$ & SFD $(\mathrm{cm})$ & SCD $(\mathrm{cm})$ & FCD $(\mathrm{cm})$ & FTA $\left(\mathrm{cm}^{2}\right)$ \\
\hline $24-26$ & 13 & $3.34 \pm 0.17$ & $2.80 \pm 0.18$ & $1.60 \pm 0.17$ & $2.21 \pm 0.25$ \\
$27-28$ & 8 & $3.76 \pm 0.20$ & $3.13 \pm 0.17$ & $1.82 \pm 0.18$ & $2.82 \pm 0.23$ \\
$29-30$ & 9 & $4.13 \pm 0.26$ & $3.41 \pm 0.18$ & 3.13 & $3.27 \pm 0.32$ \\
$31-32$ & 22 & $4.35 \pm 0.25$ & $3.50 \pm 0.21$ & $2.01 \pm 0.21$ & $3.46 \pm 0.45$ \\
$33-34$ & 56 & $4.67 \pm 0.27$ & $3.82 \pm 0.26$ & $2.14 \pm 0.22$ & $4.03 \pm 0.56$ \\
$35-36$ & 24 & $4.75 \pm 0.34$ & $3.89 \pm 0.27$ & $2.17 \pm 0.24$ & $4.16 \pm 0.64$ \\
$37-38$ & 17 & $5.06 \pm 0.29$ & $4.08 \pm 0.27$ & $2.25 \pm 0.23$ & $4.47 \pm 0.50$ \\
$39-40$ & 20 & $5.13 \pm 0.27$ & $4.20 \pm 0.26$ & $2.30 \pm 0.27$ & $4.76 \pm 0.65$ \\
$41-43$ & 9 & $5.27 \pm 0.23$ & $4.35 \pm 0.19$ & $2.20 \pm 0.21$ & $4.68 \pm 0.51$
\end{tabular}

Values are mean $\pm \mathrm{SD}$

* Birth weight and HC between third and 97th percentiles. 


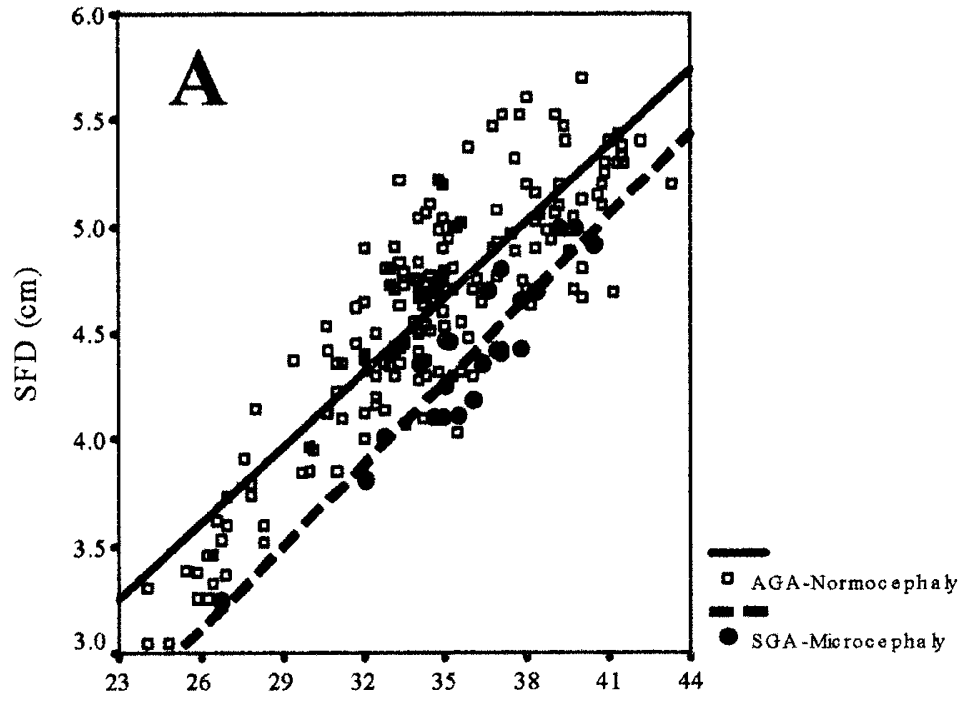

Gestational Age (weeks)

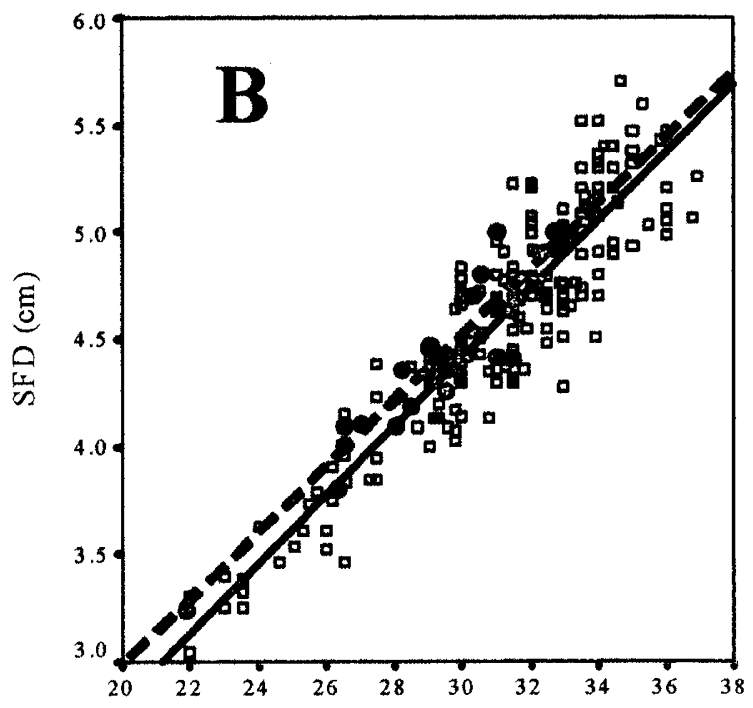

Head $\mathrm{C}$ ircumference $(\mathrm{cm})$

Figure 2. Regression lines for SFD against GA $(A)$ and $\mathrm{HC}(B)$ in the AGA-normal HC group and SGA-small HC group. Regression lines were statistically different for GA but not for HC.

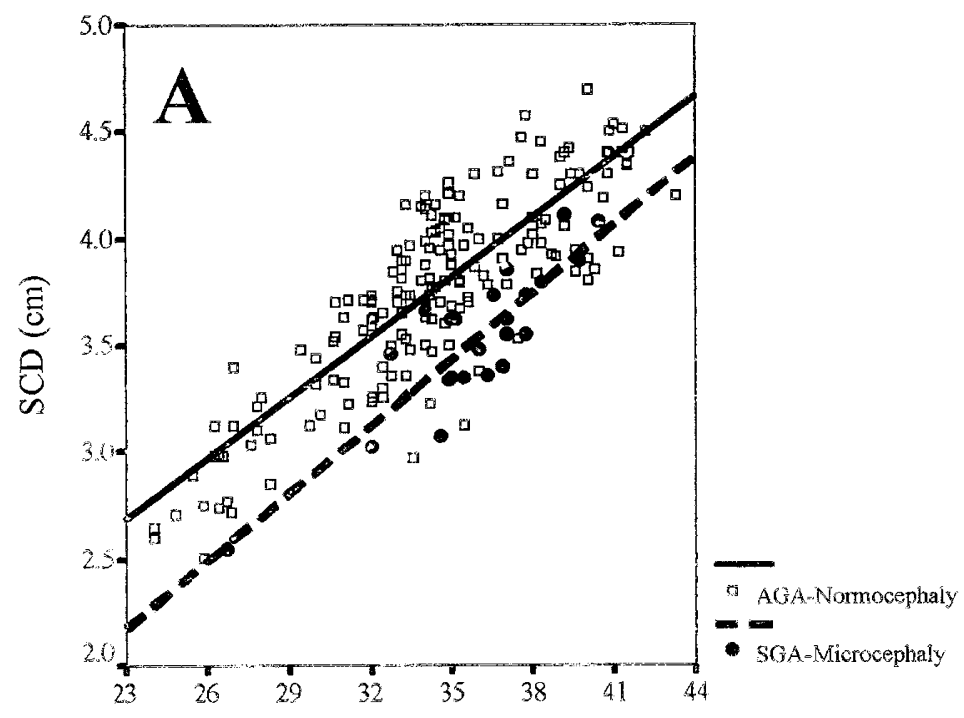

Gestational Age (weeks)

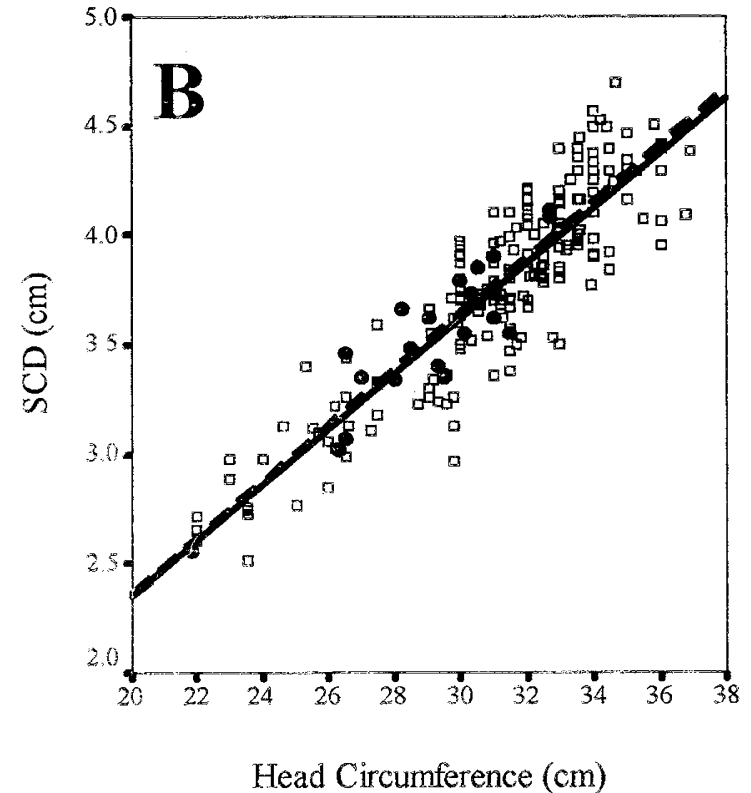

Head Circumference $(\mathrm{cm})$

Figure 3. Regression lines for SCD against GA $(A)$ and $\mathrm{HC}(B)$ in the AGA-normal HC group and SGA-small HC group. Regression lines were statistically different for GA but not for HC.

as behavioral and cognitive deficits (3). In addition, a neuropsychological study of the FL in children with mental retardation (4) showed that they display FL-related behavioral disturbances. Hence, investigation of future FL function in these neonates would be of great interest.

Using our model, the FL dimensions correlated strongly with $\mathrm{HC}$, and it appears that measurement of $\mathrm{HC}$ after birth reliably reflects the size of the FL in neonates, independent whether the third or the 10th percentile of $\mathrm{HC}$ was used as a cutoff line between normal $\mathrm{HC}$ and small $\mathrm{HC}$. The main fact is that a small head (small HC) means a small FL, which is not always the case when the fetal cerebellum is concerned (12). Our method for US assessment of the neonatal FL proved to be simple, fast, and reproducible, although it is practical only as long as the anterior fontanelle is sufficiently open. When the US coronal plane is scanned through the anterior fontanelle, the Sylvian, callosal, and frontal landmarks are easily identified.

Of the four FL dimension parameters as measured in our study, SFD and SCD showed a stronger correlation with GA, $\mathrm{HC}$, and birth weight than FTA, whereas FCD, albeit statistically significant, displayed the weakest relationship (Table 3 and Figs. 2-5). This makes SFD and SCD the optimal candidates for rapid US assessment of the neonatal FL. However, in view of the significant differences between male and female SFD and SCD, one might consider creating separate nomo- 


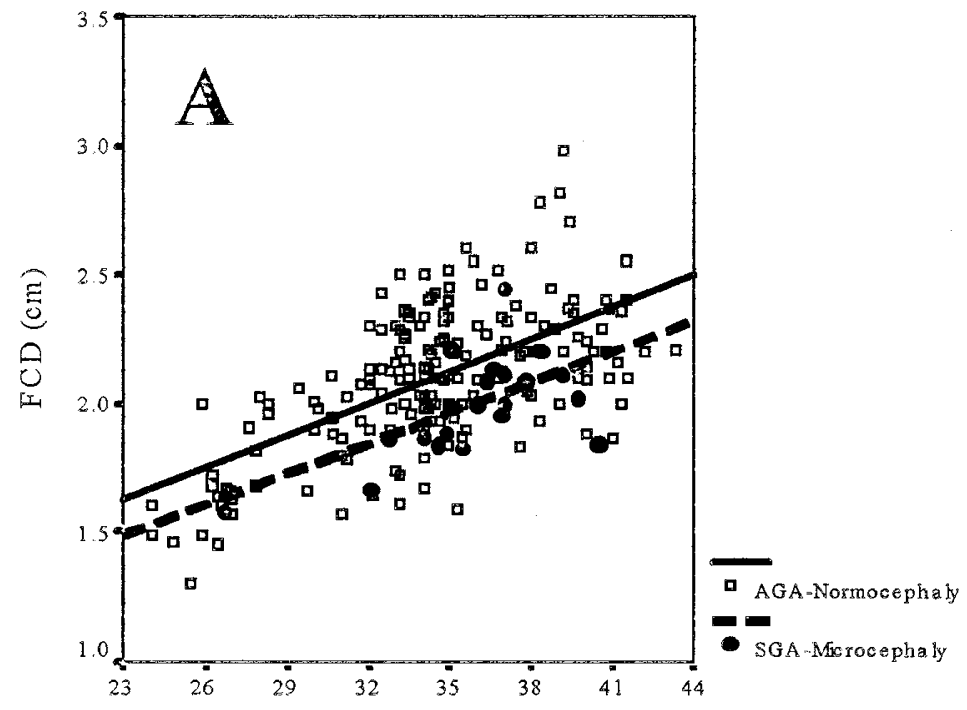

Gestational Age (weeks)

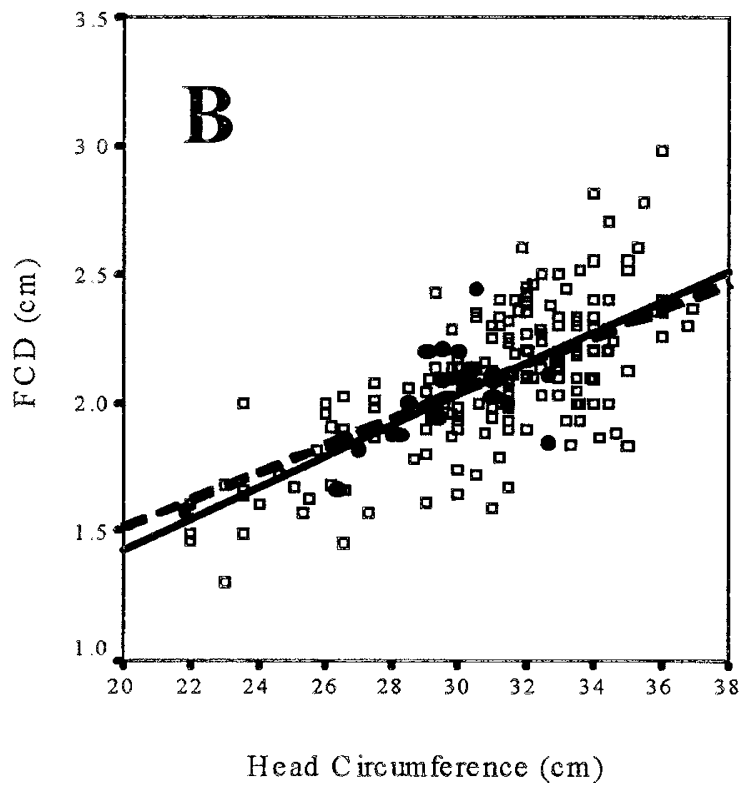

Head Circumference $(\mathrm{cm})$

Figure 4. Regression lines for FCD against GA $(A)$ and $\mathrm{HC}(B)$ in the AGA-normal HC group and SGA-small HC group. Regression lines were statistically different for GA but not for HC.

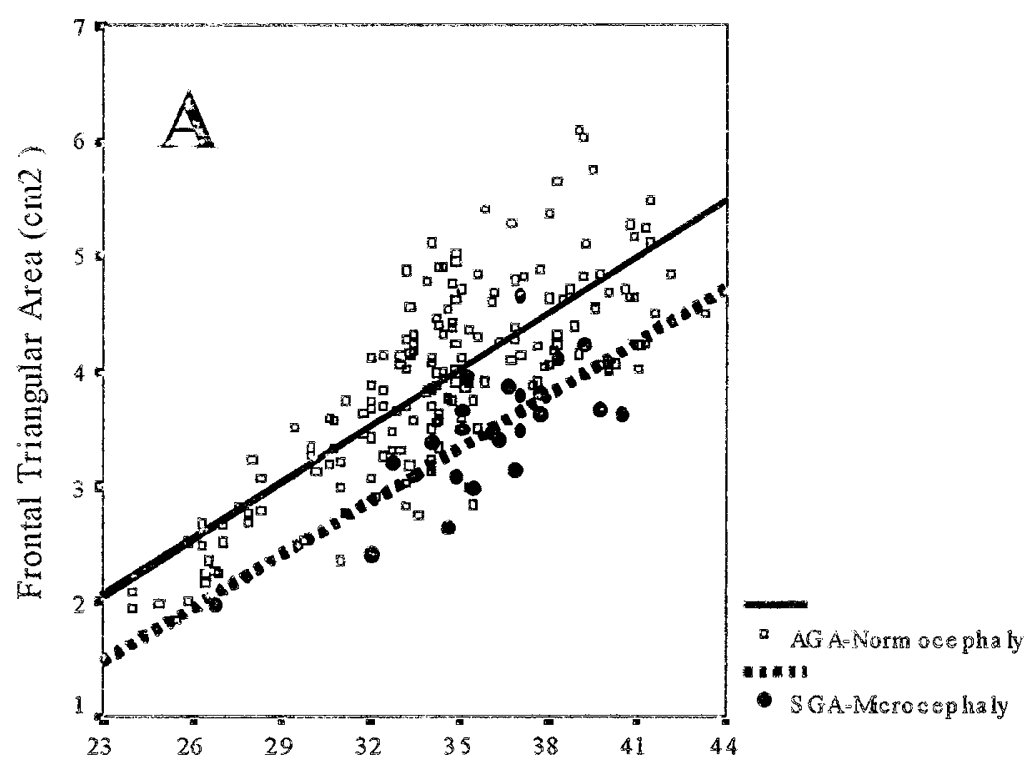

Gestational Age (weeks)

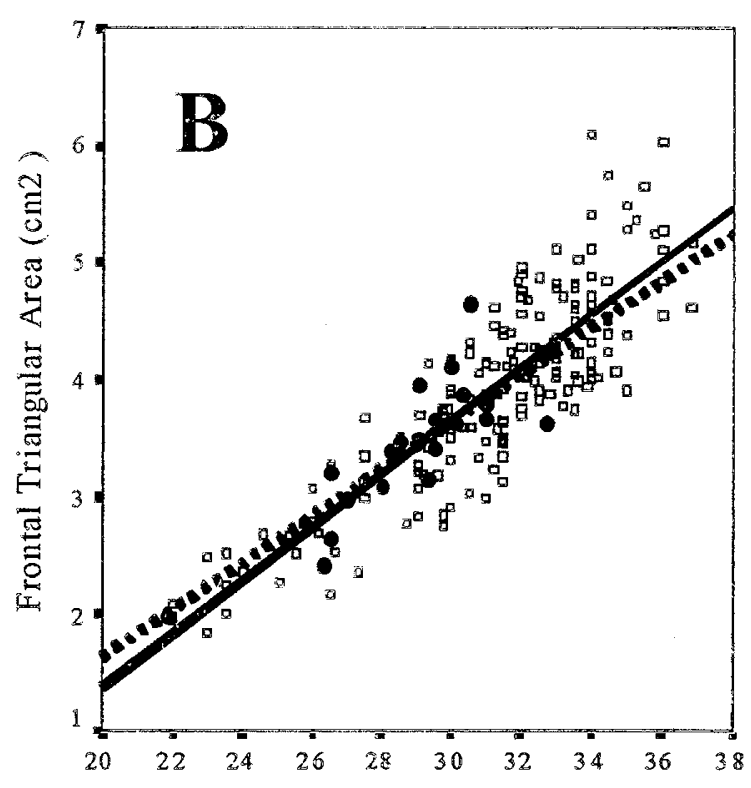

Head Circmmierence (cm)

Figure 5. Regression lines for FTA against $\mathrm{GA}(A)$ and $\mathrm{HC}(B)$ in the AGA-normal HC group and SGA-small HC group. Regression lines were statistically different for GA but not for HC.

grams of these variables, as in the standard growth curves of HC throughout infancy.

Conforming with the fact that the FL comprises about one third of the cerebral hemisphere, SGA-small HC neonates have smaller FL than their AGA-normal HC mates, and a decrease in FL size will lead to a reduction of HC. Previous reports have observed an association between SGA-small HC status and subsequent behavioral, neurologic, and intellectual impairments. Frisk et al. (22) have recently shown that IUGR that slows brain growth in utero impairs the acquisition of some cognitive and academic abilities, even when followed by good catch-up head growth after birth, whereas poor brain growth in utero followed by little or no catch-up head growth results in widespread impairments of cognitive abilities and literacy skills.

Our data may be of help for perinatologists and neonatologists when they are confronted with severe fetal IUGR and small head, or with an SGA-small HC neonate. In both conditions, parent counseling should include information regarding small $\mathrm{HC}$, small $\mathrm{FL}$, and the possibility of an increased risk for future neuro-intellectual sequelae. Moreover, a small head, i.e. small FL, in a third-trimester fetus 
Table 3. Linear growth functions of GA and HC against FL dimensions in the study groups*

AGA-normal HC neonates

GA

$$
\begin{aligned}
& R=0.867, \mathrm{SFD}=0.539+0.118 \times \mathrm{GA} \\
& R=0.846, \mathrm{SCD}=0.535+0.094 \times \mathrm{GA} \\
& R=0.613, \mathrm{FCD}=0.669+0.042 \times \mathrm{GA} \\
& R=0.789, \mathrm{FTA}=-1.704+0.163 \times \mathrm{GA}
\end{aligned}
$$

$\mathrm{HC}$

$$
\begin{aligned}
& R=0.913, \mathrm{SFD}=-0.37+0.159 \times \mathrm{HC} \\
& R=0.889, \mathrm{SCD}=-0.18+0.127 \times \mathrm{HC} \\
& R=0.691, \mathrm{FCD}=0.224+0.06 \times \mathrm{HC} \\
& R=0.856, \mathrm{FTA}=-3.17+0.23 \times \mathrm{HC}
\end{aligned}
$$

SGA-small HC neonates

GA

$$
\begin{aligned}
& R=0.926, \mathrm{SFD}=-0.271+0.13 \times \mathrm{GA} \\
& R=0.869, \mathrm{SCD}=-0.241+0.105 \times \mathrm{GA} \\
& R=0.594, \mathrm{FCD}=0.577+0.04 \times \mathrm{GA} \\
& R=0.746, \mathrm{FTA}=-2.052+0.154 \times \mathrm{GA}
\end{aligned}
$$

$\mathrm{HC}$

$$
\begin{aligned}
& R=0.924, \mathrm{SFD}=-0.079+0.153 \times \mathrm{HC} \\
& R=0.895, \mathrm{SCD}=-0.20+0.128 \times \mathrm{HC} \\
& R=0.667, \mathrm{FCD}=0.464+0.053 \times \mathrm{HC} \\
& R=0.821, \mathrm{FTA}=-2.37+0.20 \times \mathrm{HC}
\end{aligned}
$$

$* p<0.001$ for all growth functions.

Table 4. Regression analysis of perinatal variables and US dimensions of the $F L^{*}$

\begin{tabular}{cccc}
\hline & $\begin{array}{c}\text { Significantly associated } \\
\text { variables }\end{array}$ & p value & $r^{2}$ \\
\hline SFD $(\mathrm{cm})$ & GA & $<0.0001$ & 0.784 \\
& BW & $<0.0001$ & \\
& Sex & $<0.01$ & \\
SCD $(\mathrm{cm})$ & GA & $<0.0001$ & 0.758 \\
& BW & $<0.0001$ & \\
& Sex & $<0.015$ & \\
FCD $(\mathrm{cm})$ & BW & $<0.0001$ & 0.383 \\
FTA $\left(\mathrm{cm}^{2}\right)$ & GA & $<0.01$ & 0.666 \\
& BW & $<0.0001$ & \\
\hline
\end{tabular}

* Including GA, birth weight, and sex.

Abbreviation used: BW, birth weight.

with severe IUGR poses a dilemma: Will lengthening the pregnancy benefit the fetus?

Pitfalls of our study include 1) lack of neurologic and developmental follow-up of the infants studied, namely those with small HC and small FL. Hence, future prospective studies are warranted to assess the effect of small FL dimensions on future neurodevelopmental status; 2) a small number of SGA-normal HC neonates, as the US profile of the FL in these neonates is of particular interest. Unfortunately, our series included only eight such neonates, with widely spread $\mathrm{HC}$ values, and therefore their findings could not be analyzed. Future studies are needed to conclude whether the sparing of $\mathrm{HC}$ in this subgroup of SGA neonates holds true also for their FL size; 3) a small sample size of neonates born before $30 \mathrm{wk}$ of gestation, which renders creating separate nomograms for male and female infants unfeasible; and 4) we measured only the dimensions of the left FL; however, it would be worthwhile comparing the dimensions of the left and right FLs of neonates while referring to actual hemispheric dominance as it is delineated later on in early childhood.

\section{CONCLUSIONS}

We conclude that, using our sonographic model, neonatal FL dimensions are easily assessed. These FL measurements correlated strongly with $\mathrm{HC}$, and increased significantly between 24 and 43 wk of gestation. The SFD and SCD appear to be the most appropriate candidates for routine US assessment of the neonatal FL. We speculate that early severe fetal IUGR with small HC probably implies growth restriction of the FL in the fetus.

Acknowledgments. The authors thank Professor Moshe Berant and Jamal I. Makhoul for their helpful advice and assistance in the preparation of this manuscript.

\section{REFERENCES}

1. Lawson JA, Vogrin S, Bleasel AF, Cook MJ, Bye AM 2000 Cerebral and cerebellar volume reduction in children with intractable epilepsy. Epilepsia $41: 1456-1462$

2. Oki J, Miyamoto A, Takahashi S 2000 Longitudinal study of cognitive function in two patients with focal cortical dysplasia. No To Hattatsu32:408-414

3. Mazzocco MM, Hagerman RJ, Cronister-Silverman A, Pennington BF 1992 Specific frontal lobe deficits among women with the fragile-X gene. J Am Acad Child Adolesc Psychiatry 31:1141-1148

4. Markovskaia IF, Lebedinskii VV, Nikol'skaia OS 1977 Neuropsychologic characteristics of children with retarded mental development. Zh Nevropatol Psikhiatr Im S S Korsakova 77:1858-1862

5. Winter TC, Ostrovsky AA, Komarniski CA, Uhrich SB 2000 Cerebellar and frontal lobe hypoplasia in fetuses with trisomy 21: usefulness as combined US markers. Radiology 214:533-538

6. Persutte WH 1998 Microcephaly—no small deal. Ultrasound Obstet Gynecol 11:317318

7. Winter TC, Reichman JA, Luna JA, Cheng EY, Doll AM, Komarniski CA, Nghiem HV, Schmiedl UP, Shields LE, Uhrich SB 1998 Frontal lobe shortening in second-trimester fetuses with trisomy 21: usefulness as a US marker. Radiology 207:215-222

8. Persutte WH, Coury A, Hobbins JC 1997 Correlation of fetal frontal lobe and transcerebellar distance measurements: the utility of a new prenatal sonographic technique. Ultrasound Obstet Gynecol 10:94-97

9. Bahado-Singh RO, Wyse L, Dorr MA, Copel JA, O'Connor T, Hobbins JC 1992 Fetuses with Down syndrome have disproportionately shortened frontal lobe dimensions on ultrasonographic examination. Am J Obstet Gynecol 167:1009-1014

10. Goldstein I, Reece EA, Pilu G, O’Connor TZ, Lockwood CJ, Hobbins JC 1988 Sonographic assessment of the fetal frontal lobe: a potential tool for prenatal diagnosis of microcephaly. Am J Obstet Gynecol 158:1057-1062

11. Hiraiwa M, Nonaka C, Sekiyama M, Mishima M, Kobayashi M, Abe T, Fujii R, Yasukochi H 1986 Changes in frontal lobe size with age. A CT study in children. Acta Radiol Suppl 369:680-682

12. Makhoul IR, Goldstein I, Tamir A, Epelman M, Reece AE, Sujov P 2000 Transverse cerebellar distance in normal and growth restricted neonates. J Matern Fetal Med 9:155-160

13. Usher R, McLean F 1969 Intrauterine growth of live born Caucasian infants at sea level. J Pediatr 74:901-910

14. Hosmer DW, Lemeshow S 1989 Applied Logistic Regression. Wiley, New York, p 86

15. Harvey I, Ron MA, Du Boulay G, Wicks D, Lewis SW, Murray RM 1993 Reduction of cortical volume in schizophrenia on magnetic resonance imaging. Psychol Med 23:591-604

16. Kanemura H, Aihara M, Nakazawa S 2002 Measurements of the frontal and prefrontal lobe volumes by three dimensional magnetic resonance imaging 
scan-III. Analysis of sex differences with advanced age. No To Hattatsu 34:404408

17. Kanemura H, Aihara M, Nakazawa S 2002 Measurement of the frontal and prefronta lobe volumes in children with malnutrition by three dimensional magnetic resonance imaging scan. No To Hattatsu 34:398-403

18. Matsuzawa J, Matsui M, Konishi T, Noguchi K, Gur RC, Bilker W, Miyawaki T 2001 Age-related volumetric changes of brain gray and white matter in healthy infants and children. Cereb Cortex 11:335-342

19. Kanemura H, Aihara M, Aoki S, Hatakeyama K, Kamiya Y, Ono C, Sata Y, Nakazawa S 1999 Quantitative measurement of prefrontal lobe volume on three dimensional magnetic resonance imaging scan. No To Hattatsu 31: $519-524$

20. Semendeferi K, Lu A, Schenker N, Damasio H 2002 Humans and great apes share a large frontal cortex. Nat Neurosci 5:272-276

21. Sasaki M, Nakasato T, Goto H, Yanagisawa T, Suzuki T, Matsuda I, Fujiwara M, Hashimoto S, Saito K 1989 Normal sonographic findings of the infant temporal lobe in coronal sections. Brain Dev 11:230-235

22. Frisk V, Amsel R, Whyte HE 2002 The importance of head growth patterns in predicting the cognitive abilities and literacy skills of small-for-gestational-age children. Dev Neuropsychol 22:565-593 